\title{
Textile Waste Fibre Regeneration via a Green Chemistry Approach: A Molecular Strategy for Sustainable Fashion
}

\section{Xuantong Sun}

The University of Manchester

\section{Xi Wang}

the University of Manchester

\section{Fengqiang Su}

Qingdao University

\section{Mingwei Tian}

Qingdao University

\section{Lijun Qu}

Qingdao University

\section{Patsy Perry}

Manchester Metropolitan University

\section{Huw Ovens}

the University of Manchester

Xuqing Liu ( $\nabla$ xuqing.liu@manchester.ac.uk)

the University of Manchester https://orcid.org/0000-0001-5998-6546

\section{Article}

Keywords: textile recycling, sustainable, eco-friendly, green chemistry, waste textiles, fast fashion

Posted Date: June 11th, 2021

DOI: https://doi.org/10.21203/rs.3.rs-603956/v1

License: (c) (1) This work is licensed under a Creative Commons Attribution 4.0 International License. Read Full License 


\section{Abstract}

Fast fashion has been widely criticised for its excessive resource use and high generation of textile. To reduce its environmental impacts, numerous efforts have focused on finding sustainable and eco-friendly approaches to textile recycling. However, waste textiles and fibres are still mainly disposed of in landfills or by incineration and thereby pollute the natural environment, as there is still no effective strategy to separate natural fibres from chemical fibres. Herein, we developed a green chemistry strategy for the separation and regeneration of waste textiles at the molecular level. Cellulose/wool keratin composite fibres and multicomponent fibres were regenerated from waste textiles via ionic liquids. Our strategy attempts to reduce the large amount of waste textiles generated by the fast-developing fashion industry and provide a new source of fibres, which can also address the fossil fuel reserve shortages caused by chemical fibre industries and global food shortages caused by natural fibre production.

\section{Main}

Fast fashion has become increasingly popular due to its quick turnaround and low prices but has ushered throwaway culture into the clothing business, thereby limiting the sustainability of this industry. Every year, 150 billion garments are produced by the fashion industry ${ }^{1}$, of which $30 \%$ are never sold ${ }^{2}$. Over $50 \%$ are disposed of in under a year ${ }^{3}$, generating over 16 million tons of textile waste $4,85 \%$ of which ends up in landfills ${ }^{5}$ (occupying approximately $5 \%$ of landfill space ${ }^{6}$ ) or incinerated ${ }^{7}$ (when most of these materials could be reused). These numbers grow every year due to increasing overproduction and overconsumption, leading to resource waste, environmental contamination ${ }^{8}$, and potential threats to human health from microfibres found in rivers, oceans and drinking water, which could bioaccumulate via the food chain ${ }^{9-11}$.

To prolong the practical service life of fashion textiles and achieve environmental and resource sustainability, increasing textile reuse and recycling is urgently needed. Textile reuse and recycling routes, including both closed-loop and open-loop routes, can be divided theoretically into several steps ${ }^{12}$. Garments can be reused by transferring them to new owners through donating, swapping and reselling ${ }^{13}$ and, for those obsolescent ones, even upcycling by adding valuable features and remodelling ${ }^{14}$. When a garment is no longer suitable for wear, its fabric can be recycled into new products, such as upholstery, low-grade blankets, industrial rags and insulation materials ${ }^{15}$. Even after a fabric is disassembled, the remaining fibres can be isolated for potential use in carpet padding, thermal insulation ${ }^{16-18}$ and acoustic sound absorbers ${ }^{19,20}$. However, the ideal closed-loop route, in which materials are infinitely and effectively cycled without further energy consumption and pollution, cannot yet be achieved in practice. As fibres are shortened and degraded in each round of recycling, they have an estimated lifetime of 7-9 (plastic fibres) and 4-6 (cellulose fibres) reuses and recycles ${ }^{21,22}$, after which the textiles will eventually end up in landfills or incinerated, thereby polluting the natural environment. 
Despite the high production of textile wastes, their recycling rates remain low: only $15 \%$ of textile waste was collected and sorted for recycling in 2015 , and 1.1 million tonnes were lost during the process ${ }^{23}$. Most recycled textiles cascade to other industries and are downcycled into lower-value applications ${ }^{3}$.

Given the current low recycling rates, solving the problem of fashion waste and improving environmental and resource sustainability are left to material scientists. From a material perspective, the current major barrier hindering textile recycling is the lack of technologies for sorting and separation ${ }^{12}$. Most synthetic fibres, such as polyesters, can be reused easily by melt spinning when pure fractions of fibres can be isolated ${ }^{24,25}$. However, most fabrics are made of different kinds of fibres ${ }^{12}$, such as cotton/polyester and wool/polyester blends, which could be difficult or nearly impossible to isolate once blended ${ }^{26}$. Such blends cannot be simply recycled as a whole by melt spinning since natural fibres are mostly thermoset; therefore, the $\beta$-sheet nanocrystallites in the structure would be degraded prior to melting when subjected to thermal processing ${ }^{27}$. Thus, the critical question is how to separate natural fibres from synthetic fibres. Traditional methods for the regeneration of natural fibres usually employ concentrated acidic or alkaline solutions, which destroy the polymer structures of fibres, rendering them unusable as fibres in textiles and fashion. Moreover, these chemical processes unavoidably introduce hazardous substances and have been strictly prohibited in some countries ${ }^{28,29}$. As we cannot cause one environmental problem while solving another, finding a green and sustainable process for waste blended fibre separation will benefit the sustainable development of the fashion industry and reduce pollution, such as that by ubiquitous plastic particles. Ionic liquids (ILs) are closely associated with the green chemistry movement because of their low volatility, nonderivatisation and renewability. No hazardous byproducts or gas discharge are produced in reactions involving ILs. Furthermore, ILs are capable of dissolving recalcitrant natural biopolymers with massive inter- and intramolecular hydrogen bonds, such as cellulose ${ }^{30,31}$ and wool ${ }^{32,33}$, which cannot be recycled via melt spinning. To address the issues with the current status of textile waste, we designed two ILs, [BMIM][CI] and [MMIM][DMP], to separate textile waste blends and extract natural polymers from blended fabrics, leaving polyester unchanged, and successfully spun the natural polymers into pure cellulose and cellulose/wool keratin $(w / w=1 / 1)$ composite fibres. The obtained cellulose/wool keratin composite fibres have a unique morphology, with wool keratin microspheres adhered on the surface, that is different from the morphologies reported in previous studies ${ }^{34-36}$, and outstanding moisture regain, which might be due to their morphology. Acrylic was also successfully dissolved in [BMIM][CI], indicating the capability of this IL to dissolve all hydrogen-bonded polymers, both natural and synthetic. The ILs can be simply recovered and reused via distillation, thus achieving green and sustainable recycling of textile waste without the use of hazardous substances.

\section{Results}

\section{Dissolution and separation of blended polymer fabrics}

Blended cotton/polyester (50\%/50\%) and wool/polyester (50\%/50\%) fabrics were separated by dissolution with ILs, leaving undissolved polyester fibres to treat via melt spinning. [BMIM][Cl] and 
[MMIM][DMP] were applied separately to evaluate the dissolution process, and three different ratios (cotton/polyester:wool/polyester $=1: 0,1: 1,0: 1$ ) of the blended fabrics were applied in the experiment. As illustrated in Fig. 2b, the surface of the blended weaving fabric had a hair-like structure arising from the cotton fibres aligned in the weft direction; this structure was disrupted by dissolution, whereas the smooth polyester fibres aligned in the warp direction remained unchanged. During dissolution, the colour of [BMIM][Cl] turned from clear yellow to dark brown.

\section{Microstructural Changes Of The Regenerated Polymers}

To analyse the chemical changes occurring during the dissolution and regeneration, ATR-FTIR spectroscopy of native polymer fabrics, regenerated cellulose, wool keratin, cellulose/keratin composite and acrylic materials was carried out, as illustrated in Fig. 3a-d. To understand the crystallinity structures and polymorphy of the native and regenerated polymer samples, X-ray diffraction measurements were employed, and the spectra are compared in Fig. 3e-h.

The spectrum of the regenerated cellulose is similar to that of the cotton fabric: no new peaks were detected. This indicates that cellulose was recovered from the IL without distinct chemical structure changes. The broad band at $3326 \mathrm{~cm}^{-1}$ is assigned to the stretching of $-\mathrm{OH}$ groups, which indicates the presence of hydrogen bonds. The signal at approximately $2890 \mathrm{~cm}^{-1}$ could correspond to $\mathrm{C}-\mathrm{H}$ stretching, and the band at $1645 \mathrm{~cm}^{-1}$ is attributed to amorphous water. The peak at $1316 \mathrm{~cm}^{-1}$ originated from $\mathrm{O}-\mathrm{H}$ bending vibrations, and the small sharp band at $899 \mathrm{~cm}^{-1}$ could be due to $\mathrm{O}-\mathrm{H}$ stretching. The strong band at approximately $1011 \mathrm{~cm}^{-1}$ is assigned to the characteristic C-O-C stretching, the intensity of which is weaker than that in the spectrum of the raw cotton fabric. The crystalline structures of cellulose can be divided into six polymorphic forms (cellulose I, II, III, IIII, IV form in nature and can be converted into the more stable cellulose II, with a three-dimensional network structure, via regeneration or mercerization ${ }^{37}$. The diffractogram (XRD) of the cotton fabric exhibits the typical pattern of cellulose $\otimes$, with peaks at approximately $14.87^{\circ}, 16.62^{\circ}, 22.78^{\circ}$ and $34.52^{\circ}$, representing crystallographic planes (-110), (110), (220) and $(004)^{38}$. The patterns of the regenerated cellulose from the ILs show peaks at $2 \theta=12.02^{\circ}, 20.00^{\circ}, 21.70^{\circ}$ and $34.52^{\circ}$, representing crystallographic planes $(1-10)$, (110), (020) and (004), corresponding to cellulose $\rrbracket^{39}$. These results indicate the transformation from cellulose $\nabla$ to cellulose $\nabla$, with rapid breaking and reforming of inter- and intramolecular hydrogen bonds among cellulose molecules during the process ${ }^{40}$. Moreover, the lower- and borderline-crystallinity peaks of regenerated cellulose reflect a decrease in crystallinity, probably due to a coagulation process that is unfavourable to cellulose crystallization ${ }^{41}$.

The spectra of the wool fabric and regenerated wool keratin show almost identical characteristic absorption bands that could be traced back to peptide bonds (-CONH), suggesting the successful recovery of wool keratin. The absorption band at $3273 \mathrm{~cm}^{-1}$ could be due to $\mathrm{N}-\mathrm{H}$ stretching from amide $\mathrm{A}$ vibrations. The strong peak observed at $1630 \mathrm{~cm}^{-1}$ corresponds to $\mathrm{C}=\mathrm{O}$ in the amide $\mathbb{\nabla}$ band, and the 
peak at $1518 \mathrm{~cm}^{-1}$ is from N-H bending and C-N stretching in amide $\rrbracket$. A weak band at $1235 \mathrm{~cm}^{-1}$ is assigned to $\mathrm{C}-\mathrm{O}$ and $\mathrm{C}-\mathrm{N}$ stretching and $\mathrm{O}=\mathrm{C}-\mathrm{N}$ and $\mathrm{N}-\mathrm{H}$ bending in amide III. Two typical crystal structures are determined from the pattern of raw wool fabric, with a peak at approximately $10.04^{\circ}$ attributed to an a-helix structure and a peak at $21.88^{\circ}$ indicating a $\beta$-sheet structure ${ }^{42}$. The XRD spectrum of the regenerated wool keratin generally matches that of the raw wool fabric except that the peaks are significantly weaker, indicating lower crystallinity and a more amorphous structure, which can improve water absorption.

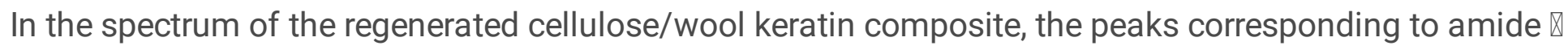
and amide II are at approximately $1638 \mathrm{~cm}^{-1}$ and $1521 \mathrm{~cm}^{-1}$, but their intensities are lower than those above due to the decreased concentration of keratin in the blend ${ }^{43}$. The peaks at $1010 \mathrm{~cm}^{-1}$ and 899 $\mathrm{cm}^{-1}$ are consistent with those in the cotton fabric. Similar to that of the regenerated cellulose, the XRD spectrum of the regenerated cellulose/wool keratin film contains peaks at $20.02^{\circ}$ and $21.83^{\circ}$, indicating that the cellulose II structure is present in the composite, and the peaks at $8.89^{\circ}$ and $12.07^{\circ}$ could be a result of the presence of wool keratin. The small and sharp peaks at $21.59^{\circ}$ and $24.00^{\circ}$ in the regenerated composite fibre are probably a result of the $\beta$-sheet structure from wool keratin and the cellulose II structure from cellulose. No new peaks appear in the FTIR spectrum of the regenerated composite in comparison with the spectra of regenerated pure cellulose and wool keratin, indicating that the macromolecular structure remains unchanged during the dissolution and regeneration; instead, bonds in the crystal structure break, resulting in polymer chain rearrangement.

The ATR spectrum of regenerated acrylic is similar to that of the raw fabric, except that it contains peaks at $3384 \mathrm{~cm}^{-1}$ and $1169 \mathrm{~cm}^{-1}$, which may correspond to the stretching of $-\mathrm{OH}$ groups and $\mathrm{C}-\mathrm{N}$ stretching, respectively. Compared to the spectrum of the original acrylic, in the spectrum of the regenerated acrylic, the major crystallinity peak at $16.96^{\circ}$ is much weaker, and the peak at $29.60^{\circ}$ is absent, which indicates that the acrylic became amorphous and that the crystal structure was damaged during recycling.

\section{Morphology of the regenerated polymer materials}

The regeneration of dissolved natural polymers can be achieved by diffusional interchange between the $\mathrm{IL}$ and a liquid bath, which is also known as the coagulation step of the wet-spinning process. Here, water was chosen for the coagulation bath, in which cellulose fibre and cellulose/wool keratin composite fibre were regenerated, and their SEM images are shown in Fig. 4a-i and Supplementary Fig. 5a-i. As shown in Fig. 4a-c, the regenerated cellulose fibre exhibits a smooth and even surface with a streaky appearance, of which the fibrillar texture was formed by the polymer chains of D-glucose units lying alongside each other ${ }^{44}$. Despite the consistency of the regenerated cellulose fibre morphology with that reported in previous studies ${ }^{34}$, it is interesting that the morphology of the regenerated cellulose/wool keratin composite fibre illustrated in Fig. $4 \mathrm{~d}-\mathrm{i}$ is significantly different from that reported previous studies ${ }^{34-36}$. Two types of cellulose/wool keratin composite fibres with the same blend ratio of $2.5 \mathrm{wt} \% / 2.5 \mathrm{wt} \%$ were obtained via wet spinning from [MMIM][DMP], presenting a similar fibre diameter but distinct morphology 
as a result of the different spinning speeds. The morphology of the composite fibre regenerated at a lower spinning speed is demonstrated in Fig. 4d-f and Supplementary Fig. 5a-c, showing tightly packed globular microparticles covering the surface, while the fibre spun at a higher spinning speed, shown in Fig. 4g-i and Supplementary Fig. 5d-i, exhibits a fibril structure along the spinning direction and 'globules' growing from the gaps. Both types of fibres display a phase-separated structure. Referring to previous studies, we found a similar globular structure in regenerated wool keratin particles and silk fibres ${ }^{27,45-48}$, thus indicating that the microspheres on the surface of the composite fibres are polymeric micelles selfassembled by wool keratin and the fibril structure was formed by cellulose (Fig. 4j).

This unique morphology may be explained by the amphiphilicity of wool keratin and the rearrangement of its chain folding. During dissolution, the breaking of disulfide bridges and hydrogen bonds is oriented by the IL, leading to the disruption of the 3D structure of wool keratin and the unfolding of the a-helix. The resultant amorphous polypeptide chain is amphiphilic, consisting of both hydrophobic and hydrophilic amino acids with their side chains extending outward, potentially enabling the formation of micelles in water. The main driving force for micelle formation, a thermodynamic process, is the minimisation of the interfacial free energy of the polymer-water system ${ }^{49}$. When the spinning dope of dissolved polymers is added to water, the polymer concentration rapidly increases and reaches the critical micelle concentration (CMC), thus inducing the self-assembly of wool keratin. As illustrated in Fig. 4k, the polypeptide chains tend to coil and aggregate once spun into the water and eventually self-assemble into a spherical structure, with the main chains bound to each other through intermolecular disulfide bonds. The R-groups from hydrophobic amino acids clump into the centre to form the core, while the hydrophilic groups from polar amino acids shield the inside from contact with water, thereby reducing the interfacial free energy to attain the most stable state. Meanwhile, cellulose, as an unbranched molecule, is regenerated from randomly oriented D-glucose subunits back to aligned polymeric chains along the spinning direction, which make up the fibril structure on the fibre surface.

The effect of the spinning conditions on micelle formation has also been demonstrated. As shown in Supplementary Fig. 5a-c, the regenerated cellulose/wool keratin fibre obtained via a lower spinning speed presents obviously more 'globules' on its surface, which suggests that the number of distributed micelles can be controlled by the duration of the contact with water. In addition, we observed that the sizes of micelles from different parts of the same fibre differ, as the diameters of the 'globules' on the anterior part of the fibre obtained at a higher spinning speed (Supplementary Fig. $5 \mathrm{~d}-\mathrm{f}$ ) vary from 140-600 nm, similar to the fibre obtained at the lower speed, while the sizes of the 'globules' on the posterior part (Supplementary Fig. $5 \mathrm{~g}-\mathrm{i}$ ) range between $100-300 \mathrm{~nm}$. This could be explained by the principle of the wetspinning process and the properties of ILs, as the filament is formed by diffusional interchange with coagulation. When ILs diffuse into water, the surface tension of the water decreases due to the addition of this surfactant, thus limiting further formation of keratin micelles.

\section{Mechanical Properties Of Regenerated Polymer Fibres}


According to previous studies $34,43,50$, the poor mechanical properties of wool keratin have limited its potential application; the addition of cellulose may enhance its mechanical strength. The practical application of the regenerated cellulose and cellulose/keratin composite fibres was studied, and the stress-strain curves are shown in Fig. 5a-c. Overall, the regenerated cellulose showed better mechanical properties than the regenerated cellulose/wool keratin composite, consistent with previous reports. The rate of moisture regain by the various fibres at $20^{\circ} \mathrm{C}$ and $25 \%$ relative humidity (RH) is given in Fig. $5 \mathrm{~d}$. Both regenerated fibres showed higher moisture regain than raw cotton and wool fibre. This higher moisture regain reflects better comfortability for wearing and is probably the result of lower crystallinity and more amorphous structure formation after regeneration. The moisture regain rate of wool keratin composite fibre is highest, reaching $18 \mathrm{wt} \%$, which may have also resulted from the increased specific surface area due to micelle structure formation.

In addition to fibres, we demonstrated the application of other regenerated cellulose/keratin composite products in the form of cyclic annular hydrogels (Fig. 5f).

\section{Conclusion}

In this study, a simple method was applied to convert waste textiles into regenerated materials. We utilised two ILs, [BMIM][CI] and [MMIM][DMP], to separate natural fibre/polyester fabrics, which achieves not only blended-fabric separation, providing a feasible method of textile recycling at scale, but also complete recycling of waste textiles and cyclic utilisation of solvents. Two types of blended fabrics, cotton/polyester and wool/polyester blends, were first dissolved by the ILs. The remaining solid polyester could be simply recycled by melt spinning, while the extracted cellulose and wool keratin could be regenerated via wet spinning.

Several types of natural polymeric products were successfully regenerated using water for coagulation, including regenerated cellulose microspheres, cellulose fibre, freeze-dried wool keratin hydrogel and cellulose/wool keratin composite fibre. We also used [BMIM][Cl] to dissolve and regenerate acrylic fabric, which became hard and fragile after regeneration and will need further study for application.

Cellulose/wool keratin composite fibres were successfully regenerated for the first time via [MMIM][DMP] and presented a significantly different morphology from that in previous reports. Microspheres were clearly observed adhered on the surface along the direction of injection, which could indicate a wool keratin component in the mixture. Such regenerated composite fibres showed good moisture regain and suitable mechanical properties, supporting their wearability.

\section{Declarations}

\section{Acknowledgements}

This research was supported by the Henry Royce Institute for Advanced Materials, funded through EPSRC grants EP/R00661X/1, EP/P025021/1, and EP/P025498/1. 


\section{Methods}

\section{Ionic liquid preparation}

1-Butyl-3-methylimidazolium chloride ([BMIM][CI]) was prepared by heating a mixture of 1methylimidazole and 1-chlorobutane at a ratio of $1: 1.1$ at $80^{\circ} \mathrm{C}$ for 24 hours, with a reflux condenser fitted to reduce volatilization. The water was removed by rotary evaporation to yield 1-butyl-3methylimidazolium chloride as a pale yellow, highly viscous liquid. 1,3-Dimethylimidazolium dimethyl phosphate ([MMIM][DMP]) was prepared in a similar way but with trimethyl phosphate instead of 1chlorobutane at a temperature of $145^{\circ} \mathrm{C}$, and the liquid obtained was dark brown.

\section{Blended fabric separation}

The fabrics were first soaked in distilled water at room temperature for 72 hours to improve their solubility in the ILs and dried at $100{ }^{\circ} \mathrm{C}$ in a vacuum oven for over 2 hours before being used. Prior to dissolution, the ILs were heated under high vacuum to reduce the water concentration, and the dried fabric samples were cut into 1-2 cm rectangular pieces. Cellulose-IL mixtures $(1: 20 \mathrm{w} / \mathrm{v})$, wool keratin-IL mixtures (1:20 $\mathrm{w} / \mathrm{v})$ and cellulose/wool keratin-IL mixtures (1:1:40 w/v) were prepared by adding $10 \mathrm{wt} \%$ of the blended fabrics (cellulose:polyester $=$ wool keratin:polyester $=1: 1$ ) to IL solutions. The fabrics were separately mixed with [BMIM][Cl] or [MMIM][DMP] in a $100 \mathrm{~mL}$ round-bottomed flask with a magnetic stirrer inside and heated in an oil bath at a constant temperature of $130^{\circ} \mathrm{C}$ for 3-10 hours. The flask was fitted with a reflux condenser, kept in a fume hood, and stirred continuously. After dissolution, the mixture was filtered to remove the solid material and cooled to room temperature, yielding the polymer-IL solution.

Subsequently, the filtrate was washed with water, dried and weighed to determine the dissolution rate of the natural fibres. An acrylic-IL solution $(1: 20 \mathrm{w} / \mathrm{v})$ was prepared in a similar way by adding $5 \mathrm{wt} \%$ acrylic fabric to $[\mathrm{BMIM}][\mathrm{Cl}]$ with stirring under refluxing until complete dissolution was confirmed.

\section{Polymer regeneration}

Water was selected as a coagulating solvent for all polymer regeneration processes.

Wet spinning

Wet spinning was carried out using a simple laboratory setup. The cellulose-IL solution, wool keratin-IL solution, cellulose/wool keratin-IL solution and acrylic-[BMIM]Cl solution were degassed prior to spinning to remove any air bubbles and then loaded into a $20 \mathrm{~mL}$ syringe equipped with a pipette tip (internal diameter, $0.5 \mathrm{~mm}$ ). The mixed solution was then extruded into a water bath and dragged at a constant speed at ambient temperature. The extruded fibres were collected and dried without further stretching at room temperature for 3 days.

Microsphere regeneration 
The cellulose-IL solution was immersed in water in a $100 \mathrm{~mL}$ glass beaker at room temperature. The solution was dispersed in water for 30 minutes to remove the IL from the cellulose. After stratification for 10 minutes, the solution was filtered under vacuum, and the filtrate was dried. The obtained filtrate consisted of cellulose microspheres. The regenerated microspheres were washed 3 times with water via centrifugation to remove any retained IL and dried under vacuum for 3 days at $45^{\circ} \mathrm{C}$.

Hydrogel regeneration

The wool keratin-IL solution was cast onto a mould, degassed to remove air bubbles, cooled to room temperature and then immersed in a deionized water bath. The water was exchanged 2 times, with each bath being used for 12 hours, to remove the IL from wool keratin and thus obtain a transparent regenerated hydrogel. The regenerated hydrogel was washed with running distilled water to fully remove the IL and freeze-dried.

After the regeneration process, the ILs were recycled by distillation.

\section{Sample characterisation}

The infrared spectra and XRD patterns of the fabrics before treatment and the polymers after regeneration were measured by a Thermo Nicolet FTIR spectrometer (Nicolet 8700) and powder X-ray diffraction (PXRD) system. Images of the sample surface morphology were collected by field emission SEM (Zeiss Ultra-55). The mechanical properties of the regenerated fibres were measured with an Instron 5943. All fibre samples were cut to a length of $20 \mathrm{~mm}$ and tested at a speed of $5 \mathrm{~mm} \mathrm{~min}^{-1}$ at ambient temperature. The moisture regain by the regenerated fibres was investigated by measuring the weight of a sample oven-dried at $100^{\circ} \mathrm{C}$ for 2 hours $\left(\mathrm{W}_{0}\right)$ and the weight of a sample placed at a constant temperature of $20^{\circ} \mathrm{C}$ and relative humidity of $25 \%$ for 24 hours $\left(W_{1}\right)$. The moisture regain rate can be obtained by the following equation:

$$
\mathrm{R}=\frac{W_{1}-W_{0}}{W_{0}}
$$

\section{References}

1. EMILY FARRA. 5 Takeaways From Last Night's Future of Fashion Sustainability Panel. VOGUE (2016).

2. Hasmik Matevosyan. Overproduction: Taboo in Fashion. (2016).

3. Ellen MacArthur Foundation. A new textiles economy: redesigning fashion's future. (2017).

4. Textiles: Material-Specific Data. United States Environmental Protection Agency (2017).

5. Humes, E. Planet junk: a journey through discards. Nature vol. 575 278-279 (2019). 
6. Recycle Shoes and Clothing | Textile Recycling Programs. http://worldwearproject.com/aboutus/global-responsibility.

7. Nunes, L. J. R., Godina, R., Matias, J. C. O. \& Catalão, J. P. S. Economic and environmental benefits of using textile waste for the production of thermal energy. J. Clean. Prod. 171, 1353-1360 (2018).

8. The price of fast fashion. Nat. Clim Chang. 8, (2018).

9. Zabala, A. Ocean microfibre contamination. Nat. Sustain. 1, 213 (2018).

10. Lau, W. W. Y. et al. Evaluating scenarios toward zero plastic pollution. Science (80-). 369, 1455-1461 (2020).

11. Walker, T. W. et al. Recycling of multilayer plastic packaging materials by solvent-targeted recovery and precipitation. Sci. Adv. 6, 1-10 (2020).

12. Sandin, G. \& Peters, G. M. Environmental impact of textile reuse and recycling e A review. J. Clean. Prod. 184, 353-365 (2018).

13. Fortuna, L. M. \& Diyamandoglu, V. Optimization of greenhouse gas emissions in second-hand consumer product recovery through reuse platforms. Waste Manag. 66, 178-189 (2020).

14. Pandit, P., Nadathur, G. T. \& Jose, S. Upcycled and low-cost sustainable business for value-added textiles and fashion. Circular Economy in Textiles and Apparel (Elsevier Ltd, 2019). doi:10.1016/B978-0-08-102630-4.00005-4.

15. Anders Schmidt, David Watson, Sandra Roos, Cecilia Askham, P. B. P. Gaining benefits from discarded textiles. (Nordic Council of Ministers, 2016).

16. Briga-sá, A. et al. Textile waste as an alternative thermal insulation building material solution. Constr. Build. Mater. 38, 155-160 (2013).

17. Trajković, D., Jordeva, S., Tomovska, E. \& Zafirova, K. Polyester apparel cutting waste as insulation material. J. Text. Inst. 5000, 1-8 (2017).

18. Lacoste, C., El Hage, R., Bergeret, A., Corn, S. \& Lacroix, P. Sodium alginate adhesives as binders in wood fibers/textile waste fibers biocomposites for building insulation. Carbohydr. Polym. 184, 1-8 (2018).

19. Seddeq, H. S. \& Aly, N. M. Investigation on sound absorption properties for recycled fibrous materials. 43, 56-73 (2012).

20. Maderuelo-sanz, R., Nadal-gisbert, A. V, Crespo-amorós, J. E. \& Parres-garcía, F. A novel sound absorber with recycled fibers coming from end of life tires (ELTs). 73, 402-408 (2012).

21. Echeverria, C. A., Handoko, W., Pahlevani, F. \& Sahajwalla, V. Cascading use of textile waste for the advancement of fibre reinforced composites for building applications. J. Clean. Prod. 208, 15241536 (2019).

22. Greener way to recycle clothes passes with flying colours. https://www.nature.com/articles/d42473019-00359-2.

23. Niinimäki, K. et al. The environmental price of fast fashion. Nat. Rev. Earth Environ. 1, 189-200 (2020). 
24. Zander, N. E., Gillan, M. \& Sweetser, D. Composite Fibers from Recycled Plastics Using Melt Centrifugal Spinning. 1-13 (2017) doi:10.3390/ma10091044.

25. Shanks, R. 5. Recycled synthetic polymer fibers in composites. Green Composites (Elsevier Ltd, 2017). doi:10.1016/B978-0-08-100783-9.00024-1.

26. H\&M's Green Machine: A recycling solution? | Vogue Business. https://www.voguebusiness.com/sustainability/handms-green-machine-a-recycling-solution.

27. Guo, C. et al. Thermoplastic moulding of regenerated silk. Nat. Mater. 19, 102-108 (2020).

28. Wang, S., Lu, A. \& Zhang, L. Progress in Polymer Science Recent advances in regenerated cellulose materials. Prog. Polym. Sci. 53, 169-206 (2016).

29. Chen, J., Vongsanga, K., Wang, X. \& Byrne, N. What Happens during Natural Protein Fibre Dissolution in Ionic Liquids. 6158-6168 (2014) doi:10.3390/ma7096158.

30. Tan, X., Li, X., Chen, L. \& Xie, F. Solubility of starch and microcrystalline cellulose in 1-ethyl-3methylimidazolium acetate ionic liquid and solution rheological properties. 27584-27593 (2016) doi:10.1039/c6cp04426c.

31. Swatloski, R. P., Spear, S. K., Holbrey, J. D. \& Rogers, R. D. Dissolution of Cellose with lonic Liquids. 4974-4975 (2002) doi:10.1021/ja025790m.

32. Xie, H. \& Zhang, S. lonic liquids as novel solvents for the dissolution and blending of wool keratin fibers. 606-608 (2005) doi:10.1039/b502547h.

33. Zheng, S., Nie, Y., Zhang, S., Zhang, X. \& Wang, L. Highly Efficient Dissolution of Wool Keratin by Dimethylphosphate lonic Liquids. (2015) doi:10.1021/acssuschemeng.5b00895.

34. Silva, R. De, Wang, X. \& Byrne, N. Development of a novel cellulose / duck feather composite fibre regenerated in ionic liquid. Carbohydr. Polym. 153, 115-123 (2016).

35. Kammiovirta, K., Anna-stiina, J., Kuutti, L. \& Holopainen-mantila, U. Keratin-reinforced cellulose filaments from ionic liquid solutions. 88797-88806 (2016) doi:10.1039/c6ra20204g.

36. Lucia, L. Polysaccharide- based Fibers and Composites. (Springer Nature). doi:10.1007/978-3-31956596-5.

37. O'SULLIVAN, A. C. Cellulose: the structure slowly unravels. Cellulose 4, 173-207 (1997).

38. Liu, Z. et al. Preparation and characterization of regenerated cellulose from ionic liquid using different methods. Carbohydr. Polym. 117, 54-62 (2015).

39. Nomura, S., Kugo, Y. \& Erata, T. 13C NMR and XRD studies on the enhancement of cellulose II crystallinity with low concentration $\mathrm{NaOH}$ post-treatments. Cellulose 27, 3553-3563 (2020).

40. Chen, H., Wang, N. \& Liu, L. Regenerated cellulose membrane prepared with ionic liquid 1-butyl-3methylimidazolium chloride as solvent using wheat straw. 1634-1640 (2012) doi:10.1002/jctb.3802.

41. Zhang, H., Wu, J., Zhang, J. \& He, J. 1-Allyl-3-methylimidazolium Chloride Room Temperature lonic Liquid: A New and Powerful Nonderivatizing Solvent for Cellulose. 8272-8277 (2005) doi:10.1021/ma0505676. 
42. Wang, K., Li, R., Ma, J. H., Jian, Y. K. \& Che, J. N. Extracting keratin from wool by using l-cysteine. Green Chem. 18, 476-481 (2016).

43. Tran, C. D. \& Mututuvari, T. M. Cellulose, Chitosan and Keratin Composite Materials: Facile and Recyclable Synthesis, Conformation and Properties. (2016) doi:10.1021/acssuschemeng.6b00084.

44. Manian, A. P., Pham, T. \& Bechtold, T. 10 - Regenerated cellulosic fibers. Handb. Prop. Text. Tech. Fibres (Second Ed. 329-343 (2018) doi:https://doi.org/10.1016/B978-0-08-101272-7.00010-9.

45. Zhang, J. et al. Isolation and characterization of biofunctional keratin particles extracted from wool wastes. 246, 356-362 (2013).

46. Sharma, S. et al. Characterization of keratin microparticles from feather biomass with potent antioxidant and anticancer activities. Int. J. Biol. Macromol. 104, 189-196 (2017).

47. Jin, H. J. \& Kaplan, D. L. Mechanism of silk processing in insects and spiders. Nature vol. 424 10571061 (2003).

48. Lu, Q. et al. Silk self-assembly mechanisms and control from thermodynamics to kinetics. Biomacromolecules 13, 826-832 (2012).

49. Owen, S. C., Chan, D. P. Y. \& Shoichet, M. S. Polymeric micelle stability. Nano Today vol. 7 53-65 (2012).

50. Hameed, N. \& Guo, Q. Blend films of natural wool and cellulose prepared from an ionic liquid. 803813 (2010) doi:10.1007/s10570-010-9411-0.

\section{Figures}

\section{Reuse}

\section{Donating, swapping reselling}

\section{Fabric recycling}

\section{Rags, blankets, upcycled clothes}

\section{Fibre recycling}

\section{Polymer recycling}

Incineration

\section{Landfill}

Carpet padding, thermal insulation, acoustic sound absorbers, soil reinforcement

Synthetic fibres: melt spinning

Natural fibres: wet spinning

Recovering energy from waste to

generate heat or electricity

Safe disposal

Figure 1 

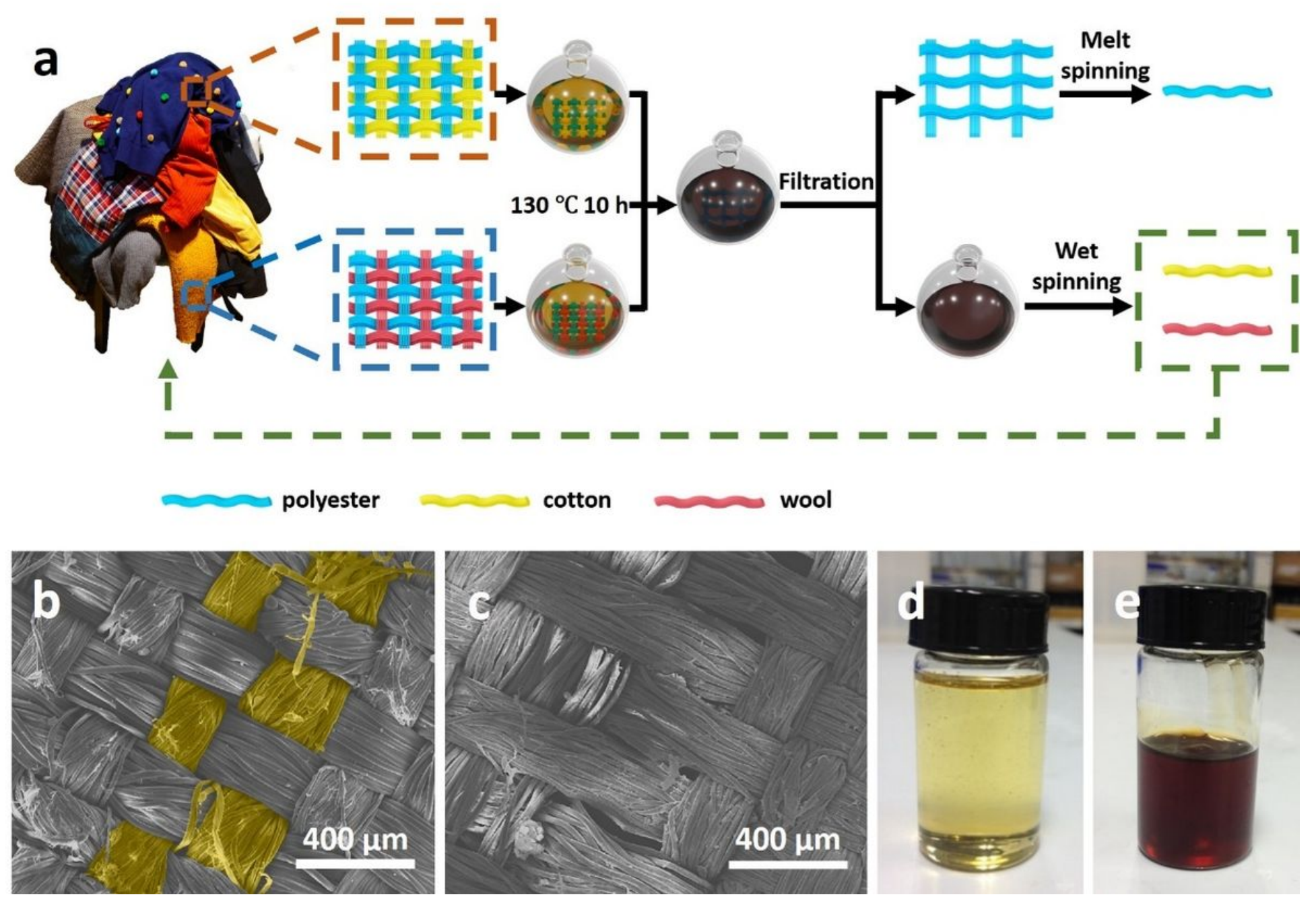

\section{Figure 2}

Performance of ionic liquids in the separation of natural polymers from polyester composite blended fabrics. a, A schematic diagram of the separation and regeneration of cotton/polyester blended fabric. b, SEM image of cotton/polyester blended fabric before dissolution. c, SEM image of the polyester fabric remaining after dissolution. $d$, Photograph of the [BMIM][Cl] ionic liquid. e, Photograph of cellulose and wool keratin dissolved in the $[\mathrm{BMIM}][\mathrm{Cl}]$ ionic liquid. 


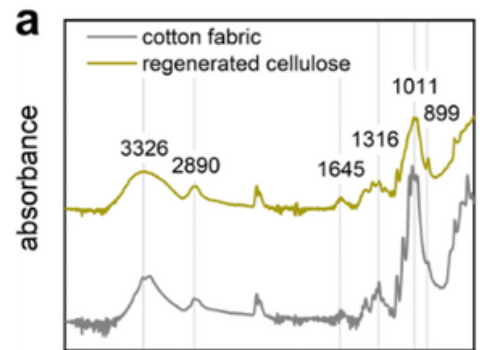

4000350030002500200015001000500 Wavenumber $\left(\mathrm{cm}^{-1}\right)$
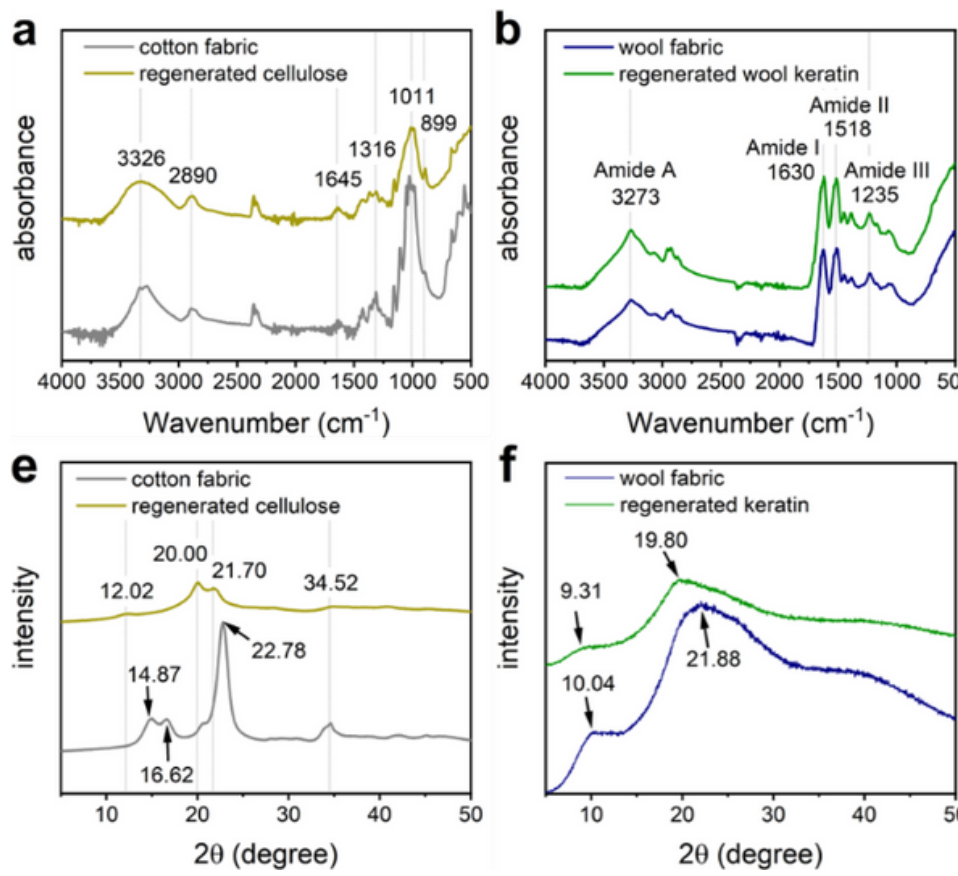

4000350030002500200015001000500 Wavenumber $\left(\mathrm{cm}^{-1}\right)$

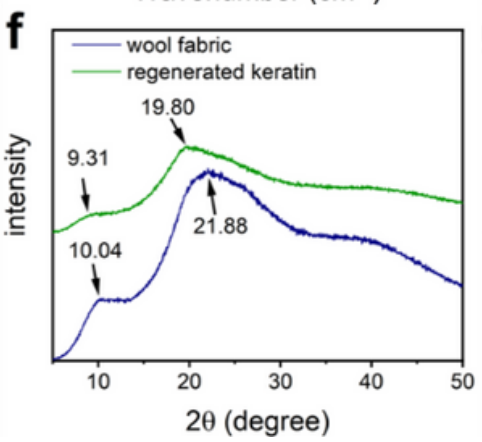

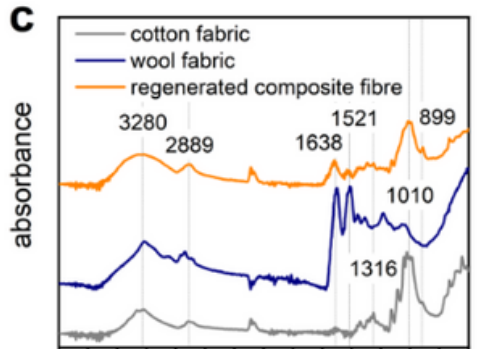

$400035003000250020001500 \quad 1000 \quad 500$ Wavenumber $\left(\mathrm{cm}^{-1}\right)$

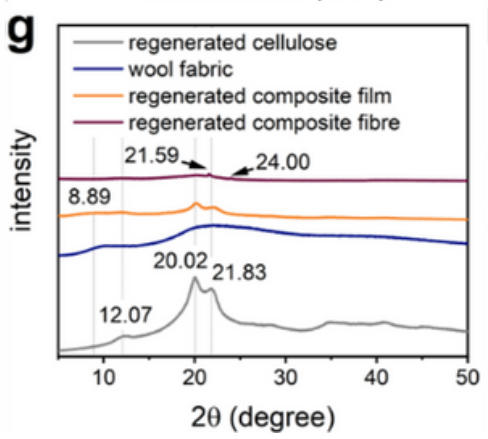

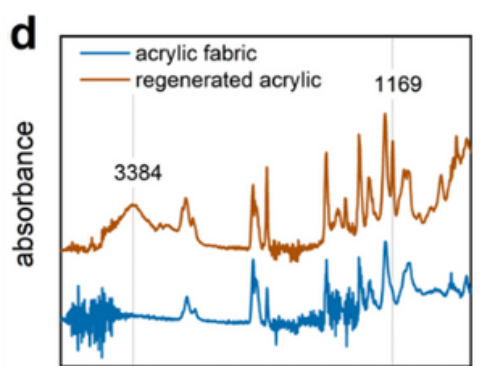

$400035003000250020001500 \quad 1000500$ Wavenumber $\left(\mathrm{cm}^{-1}\right)$

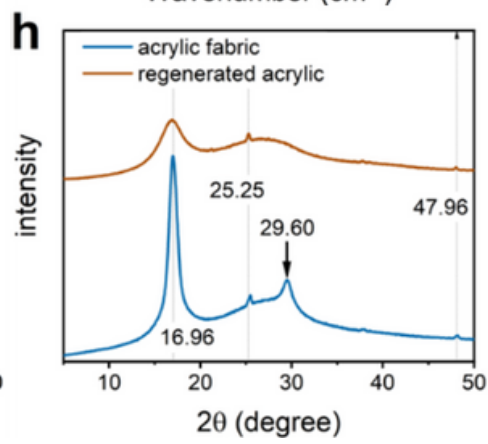

Figure 3

Characterisation of the regenerated polymer materials. FTIR spectra of (a) cotton and regenerated cellulose, (b) wool and regenerated wool keratin, (c) cotton, wool and regenerated cellulose/wool keratin composite, and (d) acrylic and regenerated acrylic. XRD patterns of (e) cotton and regenerated cellulose, (f) wool and regenerated wool keratin, (g) regenerated cellulose, wool and regenerated cellulose/wool keratin composite, and (h) acrylic and regenerated acrylic. 

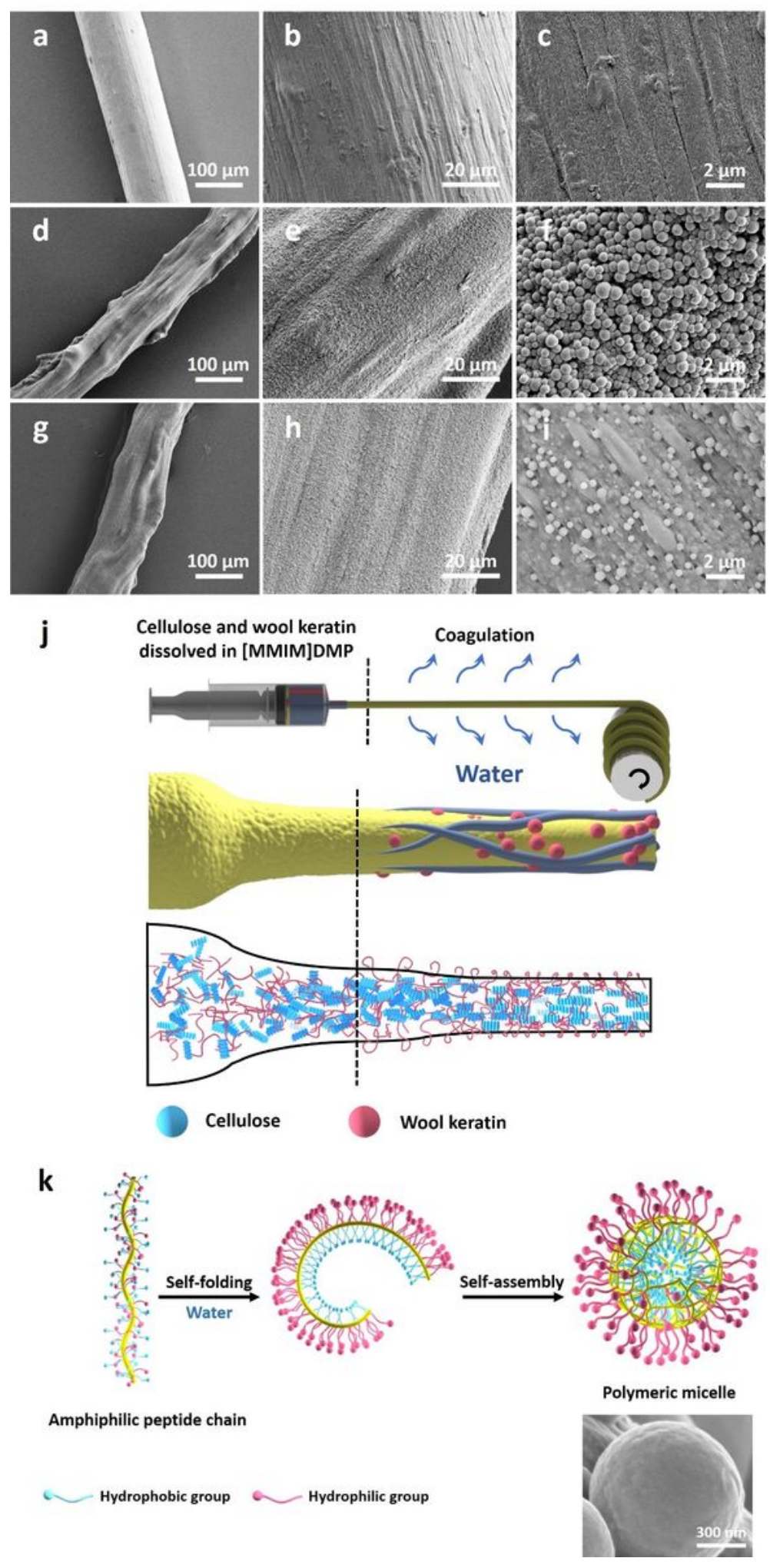

\section{Figure 4}

Morphology of the regenerated polymer fibres. a, b, c, SEM images of regenerated cellulose fibre. $d, e, f$, SEM images of cellulose/wool keratin $(w / w=1 / 1)$ composite fibre regenerated via [MMIM][DMP] at a lower spinning speed during the wet-spinning process. $\mathrm{g}, \mathrm{h}$, i, SEM images of cellulose/wool keratin $(\mathrm{w} / \mathrm{w}=1 / 1)$ composite fibres regenerated via [MMIM][DMP] at a higher spinning speed during the wet- 
spinning process. j, Schematic of the formation of the morphology of regenerated cellulose/wool keratin composite fibre. k, Self-assembly model including chain folding and micelle formation of wool keratin.
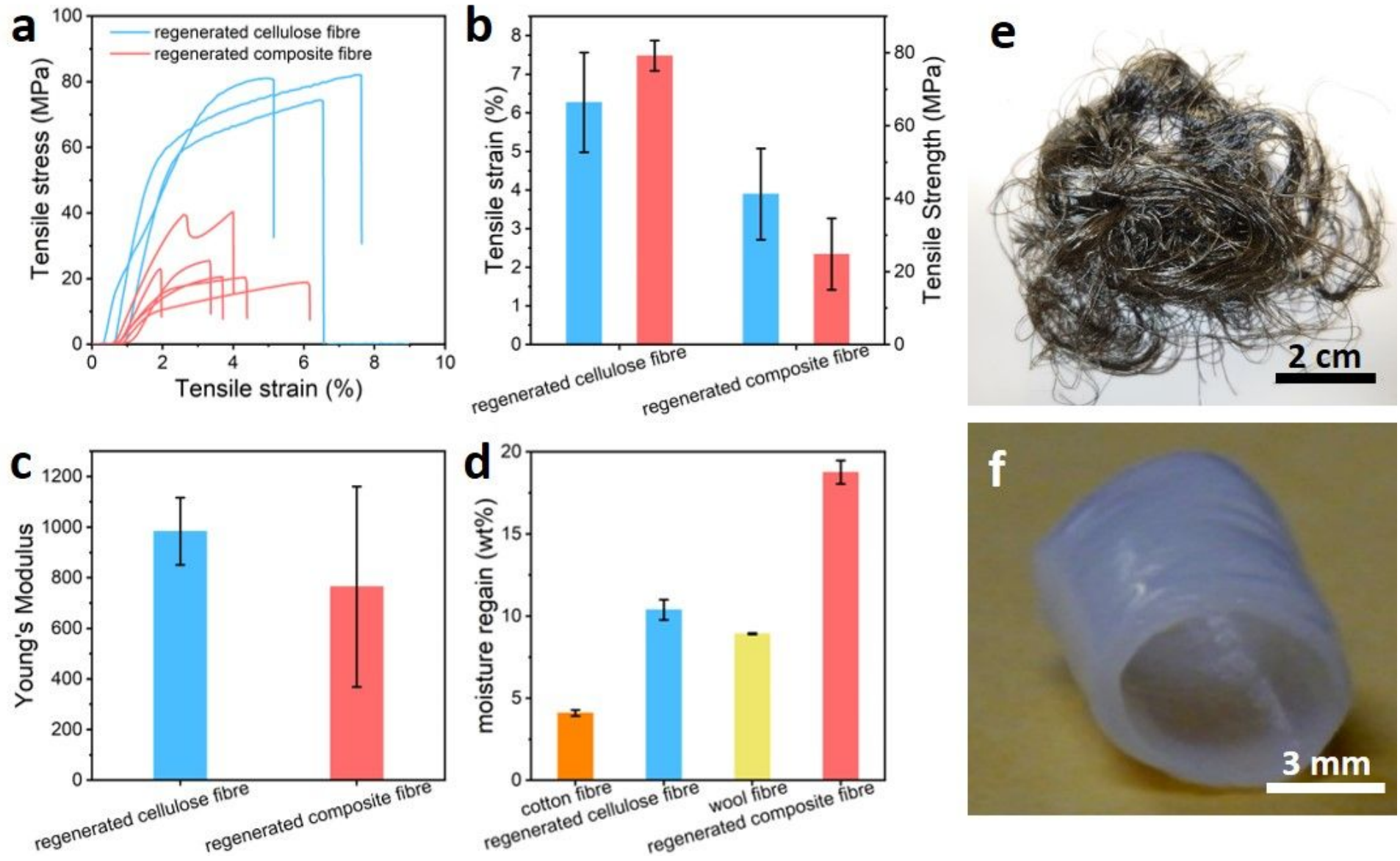

\section{Figure 5}

Mechanical properties of the regenerated polymer materials. a, Tensile strain at maximum load of the regenerated cellulose fibre and cellulose/wool keratin composite fibre. b, Tensile strength of the regenerated cellulose fibre and cellulose/wool keratin composite fibre. c, Young's modulus of the regenerated cellulose fibre and cellulose/wool keratin composite fibre. d, Moisture regain by cotton, wool, regenerated cellulose and regenerated cellulose/wool keratin composite fibres at $20^{\circ} \mathrm{C}$ and $25 \%$ relative humidity. e, A photograph of cellulose/wool keratin composite fibre regenerated via [MMIM][DMP]. $f, A$ photograph of cellulose/wool keratin hydrogel regenerated via [BMIM][CI].

\section{Supplementary Files}

This is a list of supplementary files associated with this preprint. Click to download.

- Sl.docx 